\title{
La sécurité des patients: le patient au centre
}

\section{Nadja Jenni ${ }^{a}$, Esther Kraft ${ }^{b}$, Christoph Bosshard ${ }^{\mathrm{c}}$}

${ }^{a}$ M. Sc., collaboratrice scientifique FMH, division DDQ; ${ }^{b}$ lic. rer. oec., cheffe de la division DDQ; ${ }^{c}$ Dr med., membre du Comité central de la FMH, responsable du département DDQ

Du 14 au 18 septembre 2015, la Fondation Sécurité des patients Suisse organise une semaine d'action soutenue par la FMH pour attirer l'attention sur la sécurité des patients et en rappeler l'importance. Pour la FMH, les démarches qualité et la sécurité des patients constituent deux priorités aujourd'hui indissociables du quotidien professionnel des médecins.

Une prise en charge médicale axée sur le patient revêt beaucoup d'importance pour la population, les milieux politiques et les acteurs de la santé, qui placent les patients et la sécurité toujours au centre. Par conséquent, la FMH œuvre aussi dans ce domaine et, conjointement avec d'autres acteurs, elle est un membre fondateur de la Fondation Sécurité des patients Suisse. Elle siège activement au conseil de fondation et à son comité. En Suisse, déjà très active, la sécurité des patients et les activités qualité sont depuis longtemps indissociables du quotidien professionnel des médecins. Il est donc primordial de sensibiliser à ces questions et d'informer l'opinion publique pour donner davantage de visibilité aux activités et aux démarches déjà existantes. C'est un objectif important de la semaine d'action.

La FMH soutient la Fondation depuis sa création par des aides financières ou idéelles à différents projets et va continuer à le faire:

- Poursuite du développement et du déploiement de la méthode d'analyse partenariale et évolutive des erreurs, et identification de mesures d'amélioration (projet ERA2 - Error-Risk-Analysis, 2005)

- Développement d'un cadre d'action pour une gestion interne systématique et constructive des incidents - Auteur et victime / second victim (20082010)

- Safety Hot-Spots et culture de la sécurité en médecine de premier recours (2010-2011)

- Sécurité lors du tri téléphonique en médecine de premier recours (2012-2014)

- Sécurité des patients en soins psychiatriques - Etat des lieux et plan d'action (2015)

Par ailleurs, la FMH participe à la semaine d'action par différentes activités dont notamment le présent numéro spécial du Bulletin des médecins suisses consacré à la sécurité des patients, diverses informations aux organisations de médecins et un stand sur la Waisenhausplatz à Berne, le 14 septembre 2015. Toutes ces activités sont organisées selon la devise: Le patient au centre de tout acte médical!

Créer un niveau de qualité élevé, c'est-à-dire prodiguer les bons soins au bon moment et au bon endroit, est au cœur de l'exercice de la profession de médecin. La promotion de la qualité est une évidence pour les médecins qui l'ont inscrite dans le Code de déontologie de la FMH. Dans ses activités, la FMH agit toujours en faveur des patients. Pour sa part, l'Académie suisse pour la qualité en médecine (ASQM), mise en place par la FMH, traite tous les aspects de la qualité dans le but d'obtenir des bénéfices pour les patients, les proches et les médecins.

L'ASQM supervise et soutient plusieurs projets en lien avec la sécurité des patients, dont notamment (www. asqm.ch):

- Projet-pilote «Trajectoire intersectorielle des patients atteints du cancer du côlon»

- Procédure interprofessionnelle de révision par des pairs (alliance $\mathrm{H}+$, ASDSI et FMH)

- Plateforme en ligne des initiatives qualité

- Analyse coûts-utilité des activités qualité (AQA)

- Décision partagée (Shared Decision Making), et bien d'autres encore.

La FMH continuera de s'engager auprès des politiques et des différents acteurs de la santé pour que la sécurité des patients ne soit pas au centre uniquement cette semaine. Nous vous souhaitons une lecture passionnante! 